\title{
Correction to: A phase II study of lenalidomide consolidation and maintenance therapy after autologous PBSCT in patients with multiple myeloma
}

\author{
Shin-ichi Fuchida ${ }^{1} \cdot$ Kazutaka Sunami $^{2} \cdot$ Morio Matsumoto $^{3} \cdot$ Hirokazu Okumura $^{4} \cdot$ Tohru Murayama $^{5}$. \\ Toshihiro Miyamoto $^{6}$. Eichi Otsuka ${ }^{7} \cdot$ Naohito Fujishima $^{8} \cdot$ Tohru Izumi $^{9} \cdot$ Shigehisa Tamaki $^{10}$ - Yasushi Hiramatsu ${ }^{11}$. \\ Yoshiaki Kuroda ${ }^{12}$. Chihiro Shimazaki ${ }^{1}$. Koichi Akashi ${ }^{6} \cdot$ Mine Harada $^{13}$. on behalf of the Japan Study Group for \\ Cell Therapy and Transplantation (JSCT)
}

Published online: 7 November 2018

(C) The Japanese Society of Hematology 2018

\section{Correction to: International Journal of Hematology https://doi.org/10.1007/s12185-018-2543-y}

In the original publication of this article, the "Conflict of interest" was published incorrectly. The corrected "Conflict of interest" is given below for your reading.

Conflict of interest Dr. Sunami reports grants from GlaxoSmithKline, Novartis, Janssen, Sanofi, MSD, Alexion Pharma, Daiichi-Sankyo, and Abbvie, and grants and personal fees from Ono pharmaceutical, Takeda pharmaceutical, Celgene, and Bristol-Myers Squibb. Dr. Matsumoto reports personal fees from Celgene, Janssen, Bristol-Myers Squibb, Ono Pharmaceutical, and Takeda Pharmaceutical. Dr. Okumura reports personal fees from Celgene. Dr. Murayama reports personal fees from Bristol-Myers Squibb,
Celgene, Eisai, Janssen, Kyowa Hakko Kirin, Nippon Shinyaku, Novartis Pharma, Ono Pharmaceutical, Otsuka Pharmaceutical, Pfizer, Sanofi, Siemens Healthcare Diagnostics, Sumitomo Dainippon Pharma, Taiho Pharmaceutical, Takeda Pharmaceutical, Mundipharma, Beckman Coulter, and Chugai Pharmaceutical. Dr. Miyamoto reports personal fees from Celgene. Dr. Shimazaki reports personal fees from Ono Pharmaceutical, Celgene, and Fujimoto Pharmaceutical. Dr. Akashi reports personal fees from Celgene and Janssen, grants from Nippon Kayaku, and grants and personal fees from Kyowa Hakko Kirin, Takeda Pharmaceutical, Shionogi, and Mochida Pharmaceutical. Dr. Harada reports personal fees from Celgene.

Kyushu University Hospital, Fukuoka, Japan

7 Oita Prefectural Hospital, Oita, Japan

8 Akita University Hospital, Akita, Japan

9 Tochigi Cancer Center, Utsunomiya, Japan

10 Ise Red Cross Hospital, Ise, Japan

11 Japanese Red Cross Society Himeji Hospital, Himeji, Japan

12 Hiroshima University Hospital, Hiroshima, Japan

13 Karatsu Higashimatsuura Medical Association, Karatsu, Japan 